\title{
Intelligent Systems to Predict and Diagnose Benign and Malignant Skin Lesions
}

\author{
Geetha $\mathrm{C}^{\mathrm{a}, 1}$, AparnaDarapaneni ${ }^{\mathrm{b}}$ and Lakkamaneni Chandana Manaswini ${ }^{\mathrm{b}}$ \\ ${ }^{a}$ Associate Professor, Department of CSE, R.M.K Engineering College, Chennai, \\ ${ }^{b}$ UG Scholar, Department of CSE, R.M.K Engineering College, Chennai, India
}

\begin{abstract}
The main purpose of Intelligent systems is to reason, calculate and perceive relationships and analogies. These Intelligent systems learn from experience and retrieve information from memory and provide the same to the users based onss their requirement. Currently, there is a trend for the use of intelligent systems in health informatics. The main objective of this is to improve quality, efficiency and availability of health services to people round the clock at a lower cost. Intelligent systems aim to predict and diagnose the skin cancer and abrasions based on their images. It understands the cause and thereby analyses the image based on some of the image processing techniques like patterns, anisotropic diffusion, image editing, independent component analysis and image restoration. We make use of image processing software which captures the image and then converts it to digital form and perform the required manipulations.
\end{abstract}

Keywords. Image Processing, Feature extraction, Threshold, (FCM Algorithm).

\section{Introduction}

Intelligent systems are technologically industrialized machines that perceive and respond to the problems around the world. They are embedded, internet connected computers that have the capacity of gathering and analyzing data for communication with other systems. It can find out the structure of the data and analyze the data to extract patterns and to identify strategies to act on the results of analysis. Health informatics is the act of examining, acquiring, overseeing health data and applying medical ideas related to health data innovation frameworks to enable clinicians provide better health care. Skin cancer is one of the most dangerous diseases that affects human population around the world. The primary diagnosis of this skin cancer is done visually, any skin lesions are initially detected by the clinician followed by clinical examination or can be diagnosed by a Dermatologist and then diagnosed and confirmed by Histopathological examination which is known as Biopsy. There are two kinds of techniques utilized for image processing namely digital and analog processing, Analog processing is a concept which is used for hard copies such as printouts and photos. The basics of interpretation are used while using these visual techniques.

\footnotetext{
${ }^{1}$ Geetha C, Associate Professor, Department of CSE, R.M.K Engineering College, Chennai, India Email : cga.cse@rmkec.ac.in
} 
Digital image processing helps in manipulating digital pictures using computers. The phases that are involved in digital image processing are preprocessing, Image enhancement and data extraction.

\section{Proposed Methods}

In order to detect the type of cancer mainly the following steps are needed as given in Figure 1.

\section{1 image processing}

Computer Vision plays an important role in medical image diagnosis. The tasks performed in Image processing are as follows:

- It uses filters for enhancement of an image.

- It is used mainly in order to transform the contrast, resolution, brightness and the noise level of an image.

- Typical Image processing functions are also carried out such as contouring, image sharpening and blurring.

\subsection{Image Segmentation}

In order to perform Image segmentation divide the input image into various parts called segments. As the time that is needed for the entire processing of the image will be more when compared to the processing of the partitioned image. It removes the partitions which has no information and will process only the partitions which contain the useful information. By Image segmentation we can group the partitions of the image that have similar attributes into an individual image. The types of segmentation that are mainly used:

- Region based Segmentation

- Edge detection Segmentation

- Image Segmentation based on clustering

- Threshold based segmentation

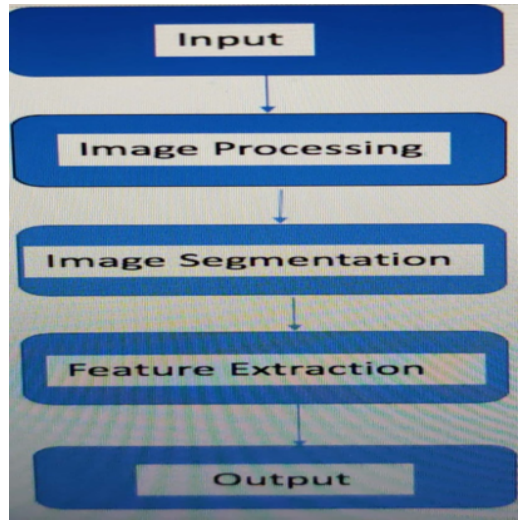

Figure 1. Flow Diagram - FCM 


\subsection{Feature Extraction}

The features mostly depend on 3D image scaling and rotation by the approach of cascade filtering in which the most expensive operations are performed only when a particular test case passes an initial test.

There are several stages in which the set of images can be arranged.

- $\quad$ Scale space extremes detection

- $\quad$ Key point localization

- Orientation assignment

- $\quad$ Key point Descriptor

The technique that is mainly used for the prediction of benign and malignant skin lesions is based on threshold segmentation. There are different types of thresholds in which the prediction can be performed. The types of threshold is given in Figure 2.

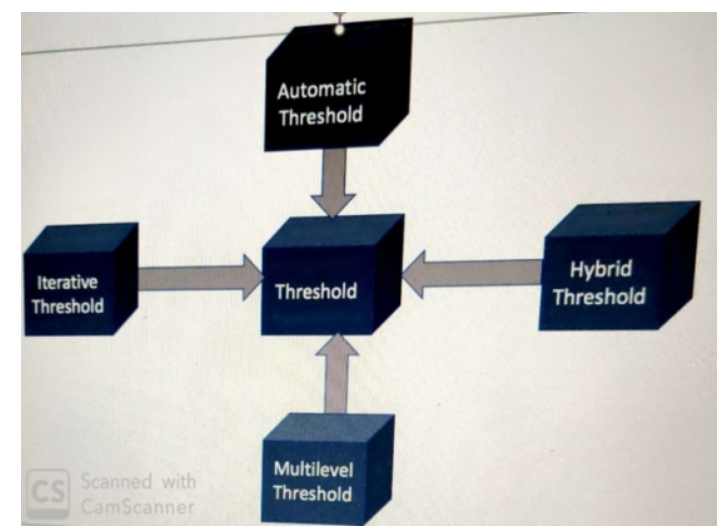

Figure 2. Types of Threshold

\subsection{Algorithm}

- Initially convert the given image into vector.

- Any number of clusters can be formed considering the vector that is derived .

- Fuzzy C uses FCM algorithm to form two or more clusters which are used in order correspond to different aspects present in the skin.

- One of the cluster can correspond to the background of the skin, the other one correspond to the cancer region and the other clusters are used to make the data that is retrieved in an efficient way.

- The result that is calculated may include some unnecessary area which is excluded and then the maximum area will be calculated.

- The result that is obtained by FCM is black and white image such that the background of the skin will be enclosed with black color and the cancer region is enclosed with white color. 


\section{Literature Survey}

Skin lesions are basically termed as lesions, dermatoses and noncancerous conditions: The cancerous conditions are given in Figure 3.

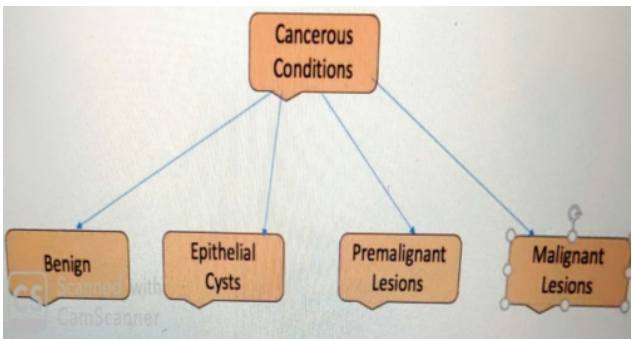

Figure 3. Cancerous Conditions

Cancer cells disobey the growth controlling signals in the body and this proliferate rapidly. Cancer cells escape death signals and achieve immortality. Imbalance between cell proliferation and cell death in cancer causes excessive growth. Cancer cells lose properties of differentiation and thus perform no function. Due to the loss of growth cells cancer cells are generally unstable. Cancer cells over run their neighboring tissue and invade locally. Cancer cell have the ability to travel from the site of origin through blood or other body fluids to other sites in the body where they colonize and establish distant metastasis.

The Lesions are of two types:

- Benign skin Lesions

- Malignant Skin Lesions

The features of both the lesions differ in the following manner as given in Figure 4.

\begin{tabular}{|c|c|c|}
\hline Festure & Benign & Malenant \\
\hline Boundaries & $\begin{array}{l}\text { Well cirumscribed and } \\
\text { encopsulated }\end{array}$ & $\begin{array}{l}\text { Poorly droumscribed and } \\
\text { Irregular }\end{array}$ \\
\hline Sumounding & Usuallycompressed & Usualy \\
\hline Size & small & Otten large \\
\hline Secondary changes & Less often & More otten \\
\hline Growth Rate & Slow & Rapid \\
\hline Local inasion & $\begin{array}{l}\text { Often compresses the } \\
\text { surrounding tissues without } \\
\text { invading or inflitrating them }\end{array}$ & $\begin{array}{l}\text { Usually inilitrates and invades } \\
\text { the adjacent tissues. }\end{array}$ \\
\hline Metastasis & Absent & Frequenty absent \\
\hline
\end{tabular}

Figure 4. Features of Benign and Malignant

The cells of both benign and malignant skin lesions are as in Figure 5. 


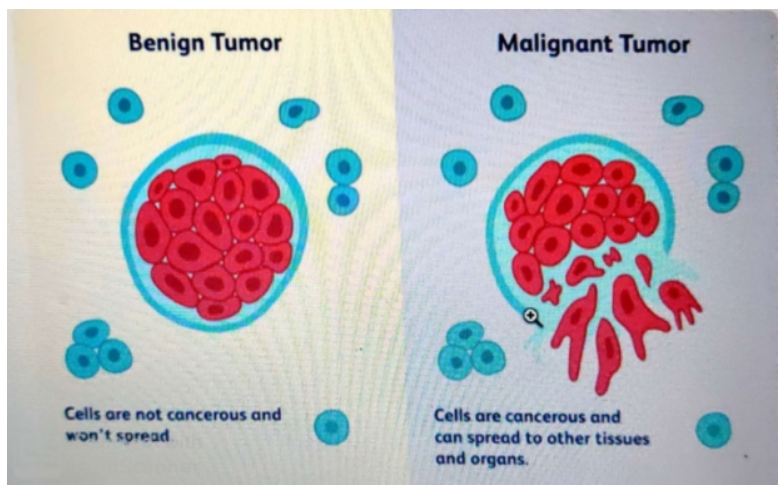

Figure 5. Cells of Benign and Malignant Lesions

\section{Result and Discussion}

The type of skin cancer is identified using image processing and Threshold based segmentation through FCM algorithm. In image processing we get the image of the affected skin area and then we apply some of the image processing techniques to get the enhanced image of the affected area. Next, we perform image analysis on the enhanced image to extract the features of the enhanced image. Later, we make use of different classifiers to identify the type of skin cancer. Finally, the type of skin cancer is recognized and diagnosed by a clinician.

\section{Conclusion}

Every year many people die of skin cancer. The rate of deaths due to skin cancer can be reduced if the proper diagnosis is done at the initial stage of the disease. Mostly these skin diseases are diagnosed visually at the beginning. So, the proper diagnosis cannot be done. That's where we make use of Threshold based Segmentation and FCM algorithm to predict the skin cancer using Image processing and to find the type of skin cancer.

\section{References}

[1] https://medicostimes.com/robbins-pathology-pdf/.

[2] https://medicostimes.com/harsh-mohan-pathology-pdf/.

[3] SnehalSalunke, Survey on Skin lesion classification and segmentation,2014.

[4] TinkuAcharya and Ajoy K. Ray, Image Processing applications and Principles, John Wiley,2005.

[5] Diagnostic Computer aided system for skin cancer Academic editor:Michael WVannier.

[6] G. Sadashivappa and SantoshAchakanalli Skin Cancer Diagnosis Using Image Processing and Implementation using Neural networks. 